The University of San Francisco

USF Scholarship: a digital repository @ Gleeson Library |

Geschke Center

2-15-1993

\title{
Magnetoresistance of Multilayered Structures for Currents Perpendicular to the Plane of the Layers
}

Horacio E. Camblong

University of San Francisco, camblong@usfca.edu

Follow this and additional works at: http://repository.usfca.edu/phys

Part of the Physics Commons

\section{Recommended Citation}

Camblong, Horacio E., "Magnetoresistance of Multilayered Structures for Currents Perpendicular to the Plane of the Layers" (1993). Physics and Astronomy. Paper 10.

http://repository.usfca.edu/phys/10 


\title{
Magnetoresistance of multilayered structures for currents perpendicular to the plane of the layers
}

\author{
Horacio E. Camblong, Shufeng Zhang, and Peter M. Levy \\ Department of Physics, New York University, New York, New York 10003
}

(Received 24 August 1992)

\begin{abstract}
While most transport measurements on multilayered structures have been done for currents in the plane of the layers, there is an emerging interest in the geometry where the currents are perpendicular to the plane of the layers. We discuss the field and current patterns in these two cases. For the latter, the elastic mean free path is not a relevant length scale; rather, it is the spin-flip mean free path that is important. In the case of currents perpendicular to the plane of the layers, one must distinguish between models which allow spin mixing of the currents and those in which the currents from the spin-up and spin-down electrons do not mix.
\end{abstract}

\section{INTRODUCTION}

Until now almost all transport measurements on metallic multilayers have been done with currents in the plane of the layers (CIP). Due to layering the current varies from one layer to another, as well as within each layer, even though a uniform electric field is applied parallel to the layers, e.g., Fig. 1 of Ref. 1. Recently there have been some novel measurements on $\mathrm{Co} / \mathrm{Ag}$ and $\mathrm{Co} / \mathrm{Cu}$ (Ref. 2) for currents perpendicular to the plane of the layers (CPP). For this geometry the current is uniform throughout, and even though a uniform electric field is applied to the layers, the actual field in the solid varies from one layer to another.

In this paper we will show (i) that for layered structures, while for CIP a one-point conductivity $\sigma(z)$ suffices, for CPP the two-point function $\sigma\left(z, z^{\prime}\right)$ is necessary, (ii) how the electric field varies from one layer to the next in the CPP geometry, and (iii) that the CPP geometry is sensitive to spin-dependent bulk scattering; in this geometry one can more readily distinguish it from interfacial scattering. ${ }^{2}$

\section{TWO-POINT CONDUCTIVITY}

The current at a point $\mathbf{r}$ is related to the electric field at $\mathbf{r}^{\prime}$ through the two-point conductivity

$$
\mathbf{j}(\mathbf{r})=\int d^{3} r^{\prime} \boldsymbol{\sigma}\left(\mathbf{r}, \mathbf{r}^{\prime}\right) \cdot \mathbf{E}\left(\mathbf{r}^{\prime}\right),
$$

where $\mathbf{E}\left(\mathbf{r}^{\prime}\right)$ is the actual electric field in the solid and $\boldsymbol{\sigma}\left(\mathbf{r}, \mathbf{r}^{\prime}\right)$ is the microscopic conductivity given by Kubo's linear-response formalism. ${ }^{3}$ We designate the layers to lie in the $x-y$ plane and the growth direction to be the $z$ axis. Due to the homogeneity in the plane of the layers, the conductivity can be written as

$$
\boldsymbol{\sigma}\left(\mathbf{r}, \mathbf{r}^{\prime}\right)=\boldsymbol{\sigma}\left(\boldsymbol{\rho}-\boldsymbol{\rho}^{\prime} ; z, z^{\prime}\right),
$$

where $\rho=(x, y)$. By taking the Fourier transform with respect to $\left(\boldsymbol{\rho}-\boldsymbol{\rho}^{\prime}\right)$, we find that Eq. (1) is written as

$$
\mathbf{j}(\mathbf{k}, z)=\int d z^{\prime} \boldsymbol{\sigma}\left(\mathbf{k} ; z, z^{\prime}\right) \cdot \mathbf{E}\left(\mathbf{k}, z^{\prime}\right) .
$$

As we are interested in cases where the fields are uniform over the layers, we take $\mathbf{k}=\mathbf{0}$ and suppress this index, namely,

$$
\mathbf{j}(z)=\int d z^{\prime} \boldsymbol{\sigma}\left(z, z^{\prime}\right) \cdot \mathbf{E}\left(z^{\prime}\right) .
$$

For fields parallel to the plane of the layers (CIP), the electric field $E(z)$ is a constant, and the current density

$$
j(z)=\sigma_{\|}(z) E,,
$$

is proportional to the one-point conductivity

$$
\sigma_{\|}(z)=\int d z^{\prime} \sigma\left(z, z^{\prime}\right),
$$

while the measured current per unit area, or average current density $\langle j\rangle=I / A$, is

$$
\langle j\rangle=\frac{1}{L} \int d z j(z)=\sigma_{\mathrm{CIP}} E,
$$

where $\sigma_{\mathrm{CIP}}$ is the global or measured CIP conductivity

$$
\sigma_{\mathrm{CIP}}=\frac{1}{L} \iint d z d z^{\prime} \sigma\left(z, z^{\prime}\right)
$$

and $L$ is the length of the sample in the $z$ direction.

For CPP the equation of continuity for the current gives, under steady-state conditions, i.e., for $\partial \varrho / \partial t=0$,

$$
\boldsymbol{\nabla} \cdot \mathbf{j}(\mathbf{r})=0 \text {, }
$$

or $j_{z}=$ const. In this geometry it is better to express the field in terms of the current density,

$$
E(z)=\int d z^{\prime} \rho\left(z, z^{\prime}\right) j\left(z^{\prime}\right)=j \int d z^{\prime} \rho\left(z, z^{\prime}\right),
$$

and the voltage drop per unit length of the sample or average electric field $\langle E\rangle=V / L$ is

$$
\langle E\rangle=\frac{1}{L} \int d z E(z)=\rho_{\mathrm{CPP}} j,
$$


where $\rho_{\mathrm{CPP}}$ is the global or measured CPP resistivity

$$
\rho_{\mathrm{CPP}}=\frac{1}{L} \iint d z d z^{\prime} \rho\left(z, z^{\prime}\right) .
$$

Therefore, while in CIP one measures the overall conductivity, in CPP it is the resistivity that is measured.

The Kubo formula yields $\sigma\left(z, z^{\prime}\right)$, and in order to find the resistivity, Eq. (12), one must either solve the integral equation

$$
\int d z^{\prime \prime} \sigma\left(z, z^{\prime \prime}\right) \rho\left(z^{\prime \prime}, z^{\prime}\right)=\delta\left(z-z^{\prime}\right)
$$

that is, invert the matrix

$$
\rho\left(z, z^{\prime}\right)=\sigma^{-1}\left(z, z^{\prime}\right) \neq\left[\sigma\left(z, z^{\prime}\right)\right]^{-1},
$$

which is unwieldy, or use a variational method to determine $\rho_{\mathrm{CPP}}$ from a knowledge of $\sigma\left(z, z^{\prime}\right)$.

\section{TWO-CURRENT MODEL}

So far we have not discussed how to incorporate spindependent scattering into the formalism of Sec. II. From the Kubo formula it follows that the total current can be regarded as coming from two spin currents, that is,

$$
j=\sum_{s=\uparrow, \downarrow} j^{s},
$$

where

$$
j^{s}=\int d z^{\prime} \sigma^{s}\left(z, z^{\prime}\right) E\left(z^{\prime}\right)
$$

and

$$
\sigma^{s}\left(z, z^{\prime}\right)=\sum_{s^{\prime}=\uparrow, \downarrow} \sigma^{s s^{\prime}}\left(z, z^{\prime}\right) .
$$

In Eq. (17), $\sigma^{s s^{\prime}}\left(z, z^{\prime}\right)$ is that part of the conductivity proportional to the current-current correlation function $\left[j^{s}, j^{s^{\prime}}\right]$, according to Kubo's formalism.

While, under steady-state conditions $(\partial \varrho / \partial t=0)$, Eq. (9) applies to the total current density, for each individual current

$$
\boldsymbol{\nabla} \cdot \mathbf{j}^{s}(\mathbf{r})=\Gamma^{s}(\mathbf{r})
$$

may be nonzero, provided that $\Gamma^{\uparrow}+\Gamma^{\downarrow}=0$.

If the internal electric field were adjusted so that one current is divergenceless, then, in general, the other current would have a finite divergence. That is, if

$$
\boldsymbol{\nabla} \cdot \mathbf{j}^{\uparrow}(\mathbf{r})=\frac{\partial}{\partial z} \int d z^{\prime} \sigma^{\uparrow}\left(z, z^{\prime}\right) E\left(z^{\prime}\right)=0,
$$

then, in general, ${ }^{4}$

$$
\nabla \cdot \mathbf{j}^{\downarrow}(\mathbf{r})=\frac{\partial}{\partial z} \int d z^{\prime} \sigma^{\downarrow}\left(z, z^{\prime}\right) E\left(z^{\prime}\right) \neq 0 .
$$

It follows that there is a mixing of the two currents, because the divergence of $j^{\downarrow}(\mathbf{r})$ implies an inflow or outflow of charge from one current channel to the other; this implies spin flip. We conclude that the same internal electric field on currents with spin up and spin down is tenable only if we allow spin-flip (spin-mixing) processes. This will be called CPP(2).

\section{INDEPENDENT CURRENTS}

In this paper we limit ourselves to multilayers with collinear magnetization configurations, e.g., ferromagnetic and antiferromagnetic configurations; this means that we can choose the quantization axis for the electron's spin along the direction parallel or antiparallel to the magnetization vectors, and that we only have two spin currents $j^{\uparrow}$ and $j^{\downarrow}$. Then, if there is no spin mixing, $\sigma^{s s^{\prime}}\left(z, z^{\prime}\right)$ is diagonal with respect to the spin indices

$$
\sigma^{s s^{\prime}}\left(z, z^{\prime}\right)=\delta_{s s^{\prime}} \sigma^{s}\left(z, z^{\prime}\right) \text {. }
$$

From the above analysis, we can see that we must introduce the condition of no-spin mixing as a constraint, e.g., by using the method of Lagrange multipliers. We do this by introducing effective internal electric fields $\mu^{s}(z)$ that make the total fields spin dependent,

$$
\mathbf{E}^{s}(z)=\mathbf{E}_{\mathrm{ext}}+\boldsymbol{\mu}^{s}(z) .
$$

When we allow this possibility, the current density for each direction of conduction electron spin is

$$
j^{s}=\int d z^{\prime} \sigma^{s}\left(z, z^{\prime}\right) E^{s}\left(z^{\prime}\right)
$$

and $\boldsymbol{\mu}^{s}(z)$ is determined by the condition that $j^{s}(z)$ is a constant, ${ }^{4}$ i.e.,

$$
\boldsymbol{\nabla} \cdot \mathbf{j}^{s}(\mathbf{r})=0
$$

or

$$
\frac{\partial}{\partial z} \int d z^{\prime} \sigma^{s}\left(z, z^{\prime}\right) E^{s}\left(z^{\prime}\right)=0 .
$$

The conductivity $\sigma^{s}\left(z, z^{\prime}\right)$ is found by using the Kubo formula for a current $j^{s}$, Eq. (23), in reacting to an electric field $E^{s}(z)$, Eq. (22). Knowing $\sigma^{s}\left(z, z^{\prime}\right)$, we can thereby find $E^{s}(z)$ and $\mu^{s}(z)$; we will call this $\operatorname{CPP}(1) .{ }^{5}$ As we will show, it leads to results that differ from $\operatorname{CPP}(2)$.

For $\operatorname{CPP}(1), j^{s}(z)$ is a constant; it is useful to invert Eq. (23) and write it as

$$
E^{s}(z)=j^{s} \int d z^{\prime} \rho^{s}\left(z, z^{\prime}\right),
$$

and the voltage drop per unit length of the sample is

$$
\langle E\rangle=j^{s} \rho_{\mathrm{CPP}}^{s},
$$

where

$$
\rho_{\mathrm{CPP}}^{s}=\frac{1}{L} \iint d z d z^{\prime} \rho^{s}\left(z, z^{\prime}\right)
$$

and

$$
\rho^{s}\left(z, z^{\prime}\right)=\left[\sigma^{s}\right]^{-1}\left(z, z^{\prime}\right) .
$$

As the voltage drop across the sample is independent of the spin direction of the conduction electrons, we find that the total current is

$$
j=\sum_{s=\uparrow, \downarrow} j^{s}=\langle E\rangle \sum_{s=\uparrow, \downarrow} \frac{1}{\rho_{\mathrm{CPP}}^{s}},
$$


and that the conductivity in $\operatorname{CPP}(1)$ is

$$
\sigma_{\mathrm{CPP}(1)}=\sum_{s=\uparrow, \downarrow} \frac{1}{\frac{1}{L} \iint d z d z^{\prime} \rho^{s}\left(z, z^{\prime}\right)} .
$$

In the $\operatorname{CPP}(2)$ model we find by following the same procedure that

$$
\rho_{\mathrm{CPP}(2)}=\frac{1}{L} \iint d z d z^{\prime} \rho\left(z, z^{\prime}\right)
$$

or

$$
\sigma_{\mathrm{CPP}(2)}=\frac{1}{\frac{1}{L} \iint d z d z^{\prime} \rho\left(z, z^{\prime}\right)},
$$

where

$$
\rho\left(z, z^{\prime}\right)=\sigma^{-1}\left(z, z^{\prime}\right)
$$

and

$$
\sigma\left(z, z^{\prime}\right)=\sum_{s, s^{\prime}=\uparrow, \downarrow} \sigma_{s s^{\prime}}\left(z, z^{\prime}\right)
$$

[see Eqs. (15)-(17)], i.e., the resistivity $\rho\left(z, z^{\prime}\right)$ is the solution to the integral equation (13). It should be emphasized that in $\operatorname{CPP}(2)$ matrix inversion of the conductivity in real space should be carried out after summation over all spin indices. Instead, for $\operatorname{CPP}(1)$ it is not possible to define $\sigma\left(z, z^{\prime}\right)$ and $\rho\left(z, z^{\prime}\right)$ without spin indices (because of the inequality of the electric fields of the two channels). Therefore, the salient difference in the conductivities of the two models is that the $\operatorname{CPP}(1)$ conductivity is the sum of the conductivities of the independent spin currents (channels), i.e., as if conduction is in parallel, while in $\operatorname{CPP}(2)$, where there is mixing of the currents in the two-spin channels, the conductivity is not expressed as simply [Eqs. (34) and (35)]. In order to obtain more explicit results, we now consider two limiting cases.

\section{LIMITING CASES}

The form of the two-point conductivity is controlled by (1) the distribution of the scatterers and (2) the intensity of the scattering. The first is dictated by the layering and is characterized by $d_{\text {in }}$, which is the repetition length of the layered pattern; the second is characterized by the mean free path $\lambda$, which represents the average of the scattering encountered over the distance $d_{\text {in }}$. There are two limits for which $\sigma\left(z, z^{\prime}\right)$ is simple: ${ }^{1}(1)$ when $\lambda \gg d_{\text {in }}$ and (2) when $\lambda \ll d_{\text {in }}$.

When $\lambda \gg d_{\text {in }}$, the conductivity is "self-averaging" and the layering is not important. This limit resembles the case in homogeneous alloys and will be referred to as such. In this case the inhomogeneities are irrelevant and translational invariance is restored, yielding a kernel

$$
\sigma\left(z, z^{\prime}\right)=F\left(z-z^{\prime}\right)=f\left(\frac{z-z^{\prime}}{\lambda}\right)
$$

whose range dependence is scaled by the average mean free path $\lambda$ (arising from the average scattering) as a characteristic length scale. In reciprocal space the twopoint conductivity is diagonal,

$$
\sigma\left(\nu, \nu^{\prime}\right)=\sigma(\nu) \delta\left(\nu-\nu^{\prime}\right)
$$

with $\sigma(\nu)$ being the Fourier transform of the real-space conductivity kernel, Eq. (36).

By writing Eq. (23) in reciprocal space, we find in the homogeneous limit that

$$
j^{s}(\nu)=\int d \nu^{\prime} \sigma^{s}(\nu) \delta\left(\nu-\nu^{\prime}\right) E^{s}\left(\nu^{\prime}\right)=\sigma^{s}(\nu) E^{s}(\nu) .
$$

For a divergenceless current $\left(j_{s}=\right.$ const $)$, this implies that $\nu=0$ and that we can use an effective constant electric field. In this case there is no distinction between $\operatorname{CPP}(1)$ and $\operatorname{CPP}(2)$; both have constant electric fields in this limit. From Eq. (37) we note that the conductivity matrix is readily inverted in reciprocal space, so that

$$
\rho^{s}(\nu)=\frac{1}{\sigma^{s}(\nu)}
$$

and that

$$
\left.\sigma_{\mathrm{CPP}(1)}\right|_{\lambda \gg d_{\mathrm{in}}}=\sum_{s=\uparrow, \downarrow} \sigma^{s}(\nu=0)=\left.\sigma_{\mathrm{CPP}(2)}\right|_{\lambda \gg d_{\mathrm{in}}}
$$

as can be seen by placing Eq. (38) in the Fouriertransformed version of Eqs. (31) and (32). From Ref. 1 it can be seen that in the homogeneous limit the CPP conductivity is identical to that in CIP, because, as mentioned before, the layering is imperceptible in this limit, and we have taken the impurity scattering to be isotropic.

When $\lambda \ll d_{\text {in }}$, which we call the "local" limit, the conductivity is a one-point function

$$
\sigma\left(z, z^{\prime}\right)=\sigma(z) \delta\left(z-z^{\prime}\right)
$$

for points $z$ and $z^{\prime}$ separated by distances $\left|z-z^{\prime}\right| \gg \lambda$. Notice that, in the local limit, for distances $\left|z-z^{\prime}\right| \lesssim \lambda$, the two-point conductivity exhibits its nonlocal structure

$$
\sigma\left(z, z^{\prime}\right)=f\left(\frac{z-z^{\prime}}{\lambda(z)}\right)
$$

whose functional form is like that of Eq. (36), but scaled with the local mean free $\lambda(z)$ [related to the one-point conductivity $\sigma(z)$ ] rather than with the average mean free path $\lambda$.

In the local limit we find differences between the two models for CPP, and both are different from the conductivity for the CIP case. By placing Eq. (41) in Eq. (23), we find that the electric field in $\mathrm{CPP}(1)$ is proportional to the inverse of the one-point conductivity $\sigma^{s}(z)$, which is precisely the case we originally made for CPP. ${ }^{5}$ In this case the spin-dependent internal electric fields (Lagrange multipliers) are

$$
\mu^{s}(z)=\frac{j^{s}}{\sigma^{s}(z)}-E_{\mathrm{ext}},
$$


where $j$ is the constant current density. In this limit the conductivity is a diagonal matrix which is easily inverted, so that

$$
\rho^{s}\left(z, z^{\prime}\right)=\frac{1}{\sigma^{s}(z)} \delta\left(z-z^{\prime}\right) .
$$

Actually, this is true only when the linear response of the system is probed over distances $\left|z-z^{\prime}\right| \gg \lambda$ (otherwise, it would be nonlocal); this is precisely the case for multilayers, when the external field is uniform, as the nonuniformity of the internal field arises from the layering (length scale $d_{\text {in }}$ ). By placing this result in Eq. (31), we find that in the local limit

$$
\left.\sigma_{\mathrm{CPP}(1)}\right|_{\lambda \ll d_{\mathrm{in}}}=\sum_{s=\uparrow, \downarrow} \frac{1}{\frac{1}{L} \int_{0}^{L} \frac{d z}{\sigma^{s}(z)}},
$$

which is just our previous result. ${ }^{5}$

For CPP(2) we find, upon placing Eq. (44) in Eqs. (32) and (34),

$$
\left.\sigma_{\mathrm{CPP}(2)}\right|_{\lambda \ll d_{\mathrm{in}}}=\frac{1}{\frac{1}{L} \int_{0}^{L} \frac{d z}{\sum_{s=\uparrow, \downarrow} \sigma^{s}(z)}} .
$$

In this limit the two cases are decidedly different; in addition, they are different from the CIP conductivity found by placing Eq. (41) in Eq. (6),

$$
\left.\sigma_{\mathrm{CIP}}\right|_{\lambda \ll d_{\text {in }}}=\sum_{s=\uparrow, \downarrow} \frac{1}{L} \int_{0}^{L} d z \sigma^{s}(z) .
$$

For CPP(2) and CIP, the local character of the conductivity, Eq. (41), makes the global conductivities, Eqs. (46) and (47), independent of the orientation of the magnetization of one layer relative to another, i.e., we note that $\sum_{s} \sigma^{s}(z)$ enters these expressions before integration. This is not the case for $\operatorname{CPP}(1)$, where, in Eq. (45), the sum over spin is taken only after one has evaluated the global resistivity for each spin channel; the latter is sensitive to average magnetization in the layered structure.

From this we conclude there is no magnetoresistance in the local limit for $\mathrm{CIP}$ and $\operatorname{CPP}(2)$; however, it does exist for $\operatorname{CPP}(1)$. By using the expressions for $\sigma^{s}(z)$ that we have previously derived, ${ }^{1,5}$ in Eq. (45), we find that in the local limit

$$
\left.\sigma_{\mathrm{CPP}(1)}\right|_{\lambda \ll d_{\text {in }}}=\frac{n e^{2}}{2 m} \sum_{s=\uparrow, \downarrow} \frac{1}{\Delta^{s}},
$$

where $\Delta^{s}=\left(\sum_{i} \Delta_{i}^{s}\right) / L$ represents the average scattering in the one-spin channel, i.e., the average of the scattering that electrons with spin direction $s$ are subject to. This expression is independent of the orientation of one layer relative to its neighbors, as long as the average magnetization $\overline{\mathbf{M}}=\sum_{i} \mathbf{M}_{i}$ is maintained. When we consider noncollinear magnetization configurations we have shown $^{6}$ that the CPP(1)-MR is the same for randomly oriented and antiferromagnetically aligned multilayered structures; both have $\overline{\mathbf{M}}=0$. However, Eq. (48) is de-

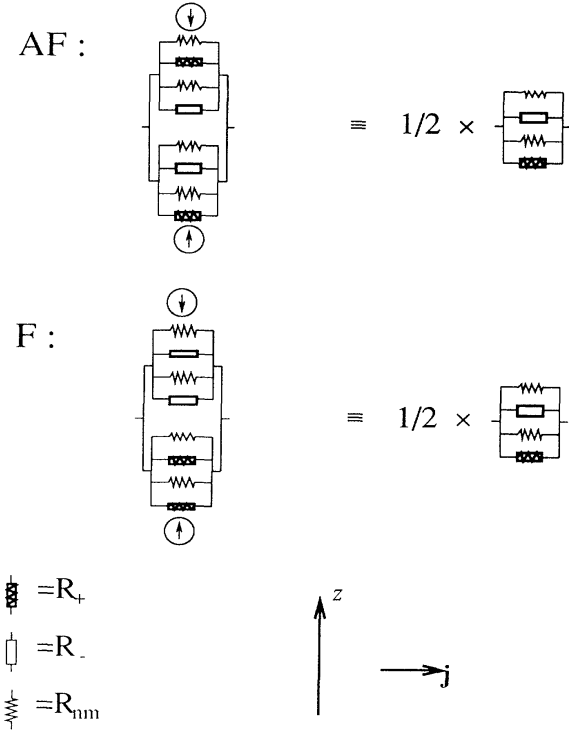

FIG. 1. Resistor network analogy for the CIP resistances of the ferromagnetic $(\mathrm{F})$ and antiferromagnetic $(\mathrm{AF})$ configurations in the local limit $\left(\lambda \ll d_{\text {in }}\right) . R_{+}, R_{-}$, and $R_{\mathrm{nm}}$ stand for the resistances of the magnetic layers for spin parallel and antiparallel to the local magnetization, and of the nonmagnetic layers. The current-density vector $\mathbf{j}$ is perpendicular to the growth direction $z$.

pendent on the average magnetization of the multilayered structure, i.e., whether $\bar{M}=0$ or $\bar{M}=M_{s}$ (saturation).

In the local limit it is meaningful to talk about the conductivity or resistivity of each layer independent of the neighboring layers. ${ }^{1}$ Thus, it is appropriate to make

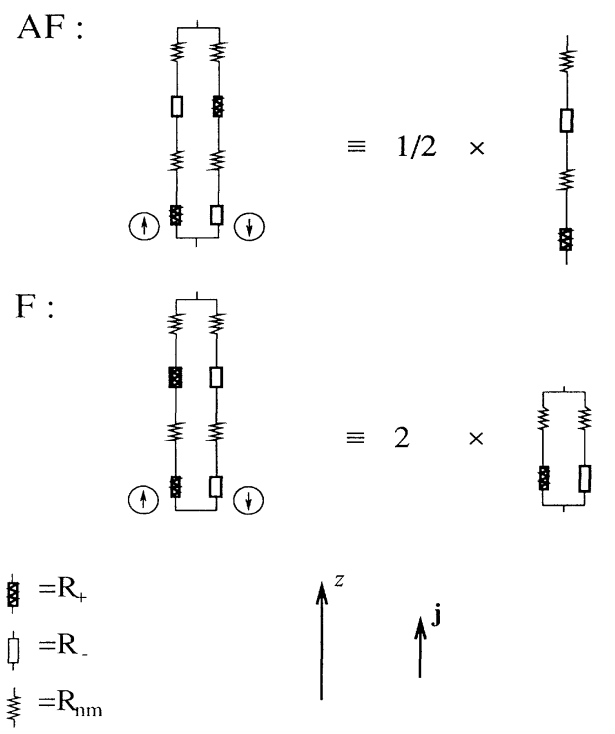

FIG. 2. Resistor network analogy for the CPP(1) resistances of the ferromagnetic (F) and antiferromagnetic (AF) configurations in the local limit $\left(\lambda \ll d_{\text {in }}\right) . R_{+}, R_{-}$, and $R_{\mathrm{nm}}$ have the same meaning as in Fig. 1 . The current-density vector $\mathbf{j}$ is parallel to the growth direction $z$. 
$\mathrm{AF}$ :

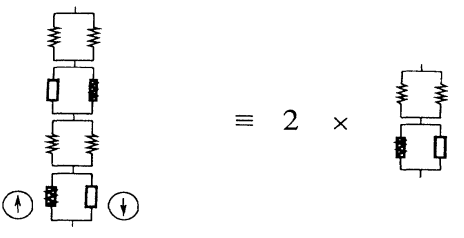

$$
\text { F : }
$$

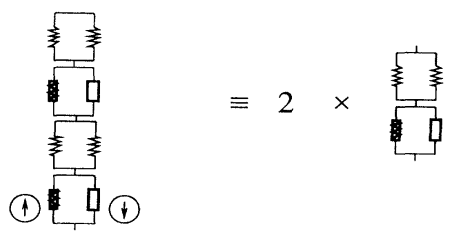

$$
\begin{aligned}
& =R_{+} \\
& =R_{-} . \\
& =R_{n m}
\end{aligned}
$$

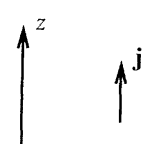

FIG. 3. Resistor network analogy for the $\mathrm{CPP}(2)$ resistances of the ferromagnetic (F) and antiferromagnetic (AF) configurations in the local limit $\left(\lambda \ll d_{\text {in }}\right) . R_{+}, R_{-}$, and $R_{\mathrm{nm}}$ have the same meaning as in Fig. 1. The current-density vector $\mathbf{j}$ is parallel to the growth direction $z$.

analogies with resistor networks to understand whether or not the resistivity changes in going from an antiferromagnetic (random) configuration to a ferromagnetic alignment of the magnetic layers. In Fig. 1 we show the appropriate network corresponding to four layers, two magnetic, two nonmagnetic, in CIP, Eq. (47). We see that the resistivities are the same for the ferromagnetic $(\mathrm{F})$ and antiferromagnetic (AF) configurations, so that there is no CIP-MR in this limit. For CPP(1), where there is no mixing of the currents (for electrons with spin up with those of spin down), the resistances of the individual layers for each spin direction are added in series, while those for the two channel are added in parallel. In Fig. 2 we note that in the ferromagnetic configuration the resistance is less in one branch than in the other, producing a "short-circuit" effect while in the antiferromagnetic configuration they are equal. Thus we have magnetoresistance for $\mathrm{CPP}(1)$. However, when the currents are mixed via spin flips, the resistors are coupled as shown in Fig. 3, and we see there is no short-circuit effect for this case, i.e., there is no magnetoresistance for $\mathrm{CPP}(2)$ in the local limit.

\section{CURRENTS AND FIELDS}

For the CIP geometry we have the same electric field in every layer, and the current is proportional to the onepoint conductivity $\sigma^{s}(z)$. Typical variations of the current across a multilayered structure have been previously depicted. ${ }^{1}$ Here we want to show that while the current is constant for CPP throughout, it is the electric field that varies. In general, to determine $E^{s}\left(z^{\prime}\right)$, it is necessary to know the two-point conductivity $\sigma^{s}\left(z, z^{\prime}\right)$; however, in the local limit $\left(\lambda \ll d_{\text {in }}\right)$, the one-point conductivity suffices, because

$$
\left.E^{s}(z)\right|_{\lambda \ll d_{\mathrm{in}}}=\frac{j^{s}}{\sigma^{s}(z)} .
$$

In Fig. 4 we show the current and field distribution across three layers (two magnetic, one nonmagnetic) for no mixing of the currents, $\operatorname{CPP}(1)$, with the condition $\sigma^{\uparrow}>\sigma^{\downarrow}$ for the magnetic layers and $\sigma^{\uparrow}=\sigma^{\downarrow}$ for the nonmagnetic. The "sum rule"

$$
\int d z E^{\uparrow}(z)=\int d z E^{\downarrow}(z)=V_{\mathrm{ext}}
$$

should be applied when one tries to understand the changes in $E^{s}(z)$ as one goes across the layers.

With spin flips, the currents $j^{s}(z)$ vary with distance on the length scale of the spin-diffusion length $\left(\lambda_{s d}\right)$, see Fig. 5. Actually, as we have not introduced spin flips in our theory, we are not able to calculate $E\left(z^{\prime}\right)$; however, we can intuit what follows. Far from the interfaces (compared to $\lambda_{s d}$ ), the electric field is unique and $E^{\uparrow}=E^{\downarrow}=E$. However, close to the interfaces the mixing of the currents for spin up and spin down is insufficient to maintain this condition. The salient difference with $\operatorname{CPP}(1)$ shows up in the current distribution; although $j=\sum_{s} j^{s}$ is constant the individual $j^{s}$ varies with distance; in fact, in the antiferromagnetic state the spin-up and spin-down currents exchange roles. One becomes smaller while the other becomes larger.
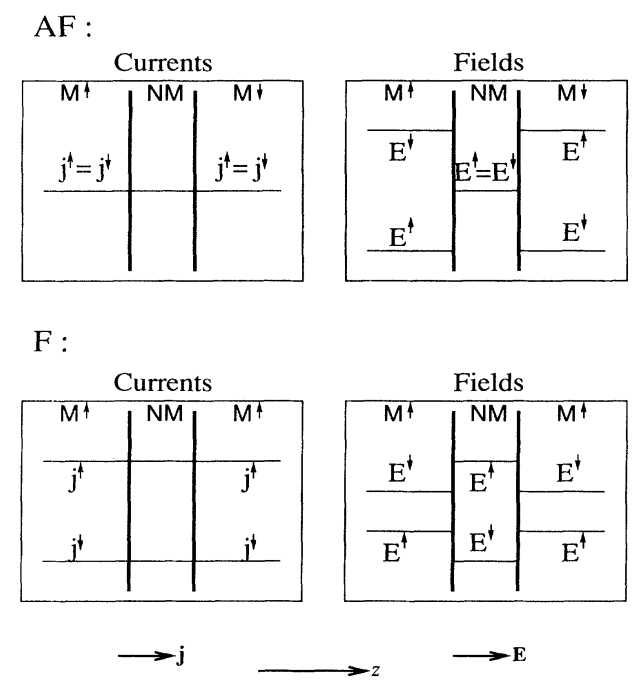

FIG. 4. Current densities $\left(j^{\uparrow}\right.$ and $\left.j^{\downarrow}\right)$ and electric fields $\left(E^{\uparrow}\right.$ and $E^{\downarrow}$ ) for both spin channels, across two magnetic layers (M) and one nonmagnetic layer (NM), for CPP(1) (no spin mixing) and in the local limit $\left(\lambda \ll d_{\text {in }}\right)$. The magnetizations of the magnetic layers are parallel $(M \uparrow$ and $M \uparrow)$ for the ferromagnetic configuration (F) and antiparallel $(M \uparrow$ and $M \downarrow$ ) for the antiferromagnetic configuration (AF). The current density $(\mathbf{j})$ and the electric field $(\mathbf{E})$ are parallel to the growth direction ( $z$ axis). It is assumed that $\sigma^{\uparrow}>\sigma^{\downarrow}$ for the magnetic layers. 

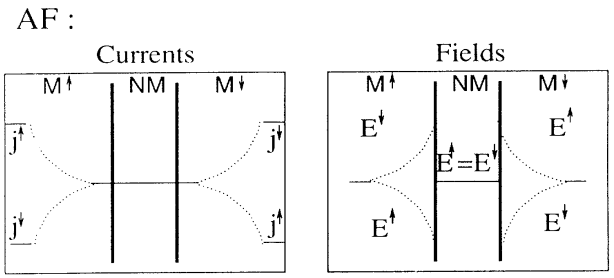

F :
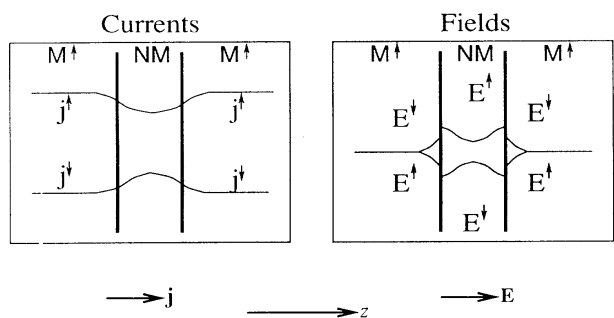

FIG. 5. Current-density $\left(j^{\dagger}\right.$ and $\left.j^{\downarrow}\right)$ and electric-field distributions $\left(E^{\dagger}\right.$ and $\left.E^{\downarrow}\right)$ for both spin channels, across two magnetic layers $(M)$ and one nonmagnetic layer (NM), in the presence of a small amount of spin mixing and in the local limit $\left(\lambda \ll d_{\text {in }}\right)$. The magnetizations of the magnetic layers are parallel $(M \uparrow$ and $M \uparrow)$ for the ferromagnetic configuration (F) and antiparallel $(M \uparrow$ and $M \downarrow)$ for the antiferromagnetic configuration (AF). The current density (j) and the electric field (E) are parallel to the growth direction ( $z$ axis). It is assumed that $\sigma^{\uparrow}>\sigma^{\downarrow}$ for the magnetic layers. For the AF configuration the dotted lines represent the current and field patterns over a length scale $\lambda_{s d}$ much larger than the thickness of the nonmagnetic layer. For the ferromagnetic configuration the relative changes in the currents and fields are small and occur near the NM layer.

This seems correct inasmuch as the majority spin current (conduction-electron spin parallel to the magnetization) is higher for $\sigma^{\uparrow}>\sigma^{\downarrow}$; however, this does not occur in $\operatorname{CPP}(1)$, because without spin flips there is no way of transferring charge from one current to the other.

The current distribution in Fig. 5 can be understood with reference to $\operatorname{CPP}(1)$, Fig. 4 , where one recognizes that in the magnetic layers the different conductivities $\sigma^{s}$ act to create differences in the currents $j^{s}$, while in the nonmagnetic layers the equality of the conductivities tends to equilibrate the currents $j^{s}$. The electric fields in Fig. 5 can also be understood with respect to $\operatorname{CPP}(1)$ by noting that, far from the interfaces (with respect to $\left.\lambda_{s d}\right)$, the electric fields $E^{s}(z)$ tend to become the same.

We conclude with some unusual predictions for the magnetoresistance for $\operatorname{CPP}(1)$. In general, we need the two-point conductivity $\sigma\left(z, z^{\prime}\right)$ and the electric fields $E^{s}\left(z^{\prime}\right)$. However, we note that in the local limit, $E^{s}(z)$ is proportional to the inverse of $\sigma^{s}(z)$, Eq. (49), and that in the homogeneous limit this is trivially satisfied as $\sigma^{s}$ is independent of position. Therefore, as an approximation, we take $E^{s}(z) \propto\left[\sigma^{s}(z)\right]^{-1}$ over the entire range of layering repeat distances $d_{\text {in }}$ relative to the mean free

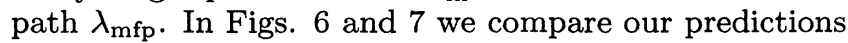
for the CPP(1)-MR with the behavior of the CIP-MR.

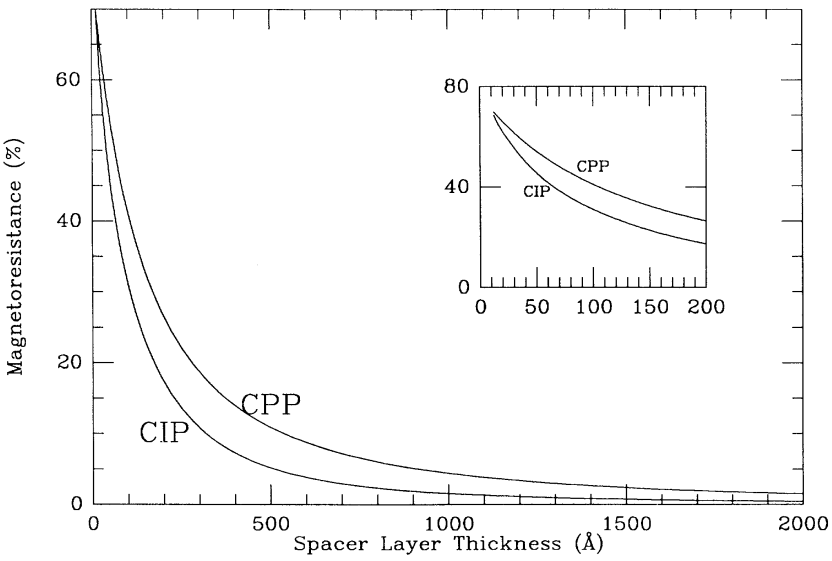

FIG. 6. Magnetoresistance vs spacer layer thickness $t_{\mathrm{nm}}$ for fixed magnetic layer thickness $t_{m}=30 \AA$. The parameters used for these curves are (see Refs. 1 and 7 for the meaning of the parameters) $\lambda_{m}=40 \AA, \lambda_{\mathrm{nm}}=200 \AA$, $\lambda_{s}=\left(t_{m}+t_{\mathrm{nm}}\right) / 0.6 ; p_{s}=0.5$ and $p_{b}=0.2$. These are the mean free paths for the magnetic and nonmagnetic layers and interface, and the ratio of spin-dependent to spin-independent scattering potentials for the interface and the bulk, respectively.

To obtain these figures we used the formulas derived in Refs. 1 and 5. The parameters we used are close to those we have found by fitting the data on CIP-MR and CPP$\mathrm{MR}$ of $\mathrm{Co} / \mathrm{Cu}$ or $\mathrm{Co} / \mathrm{Ag}$. We note the magnetoresistance behaves differently if the thickness of the magnetic layer $t_{m}$ is constant, as compared to when it varies in size so as to be equal to the thickness of the nonmagnetic layer $t_{\mathrm{nm}}$.

When $t_{m}$ is held fixed both CIP-MR and CPP-MR approach zero as $t_{\mathrm{nm}}$ increases, but for different reasons; see Fig. 6. The decrease in the CIP-MR comes from the inability of the electrons to sample more than one magnetic layer within the distance of the mean free path $\lambda_{\mathrm{mfp}}$, as the thickness of the nonmagnetic layer $t_{\mathrm{nm}}$ increases.

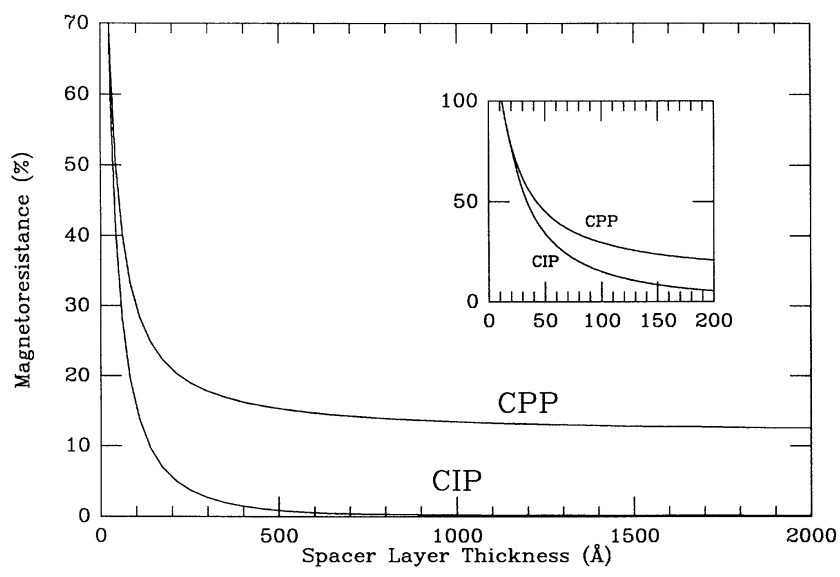

FIG. 7. Magnetoresistance vs spacer layer thickness $t_{n m}$ for equal layer thicknesses $t_{m}=t_{\mathrm{nm}}$. The parameters used for these curves are the same as in Fig. 6. 
For $\operatorname{CPP}(1)$, there is no decay from mean-free-path effects. Rather the CPP(1)-MR decreases due to the reduced spin-dependent scattering per unit length as $t_{\mathrm{nm}}$ increases.

When $t_{m}=t_{\mathrm{nm}}$, see Fig. 7, the CPP(1)-MR reaches a finite asymptote, which comes from the spin-dependent bulk scattering per unit length. This is held constant, because as we increase $t_{\mathrm{nm}}$ so we also add $t_{m}$ to maintain the equality of the two. We note from Fig. 7 that the CPP-MR with $t_{m}=t_{\mathrm{nm}}$ provides a sensitive test for the presence of spin-dependent bulk scattering. If it is not present the CPP-MR goes to zero as $t_{\mathrm{nm}}$ increases in much the same way as in Fig. 6. The curve for the CPP-MR in Fig. 7 is predicated on $\operatorname{CPP}(1)$, i.e., the assumption that all the distances $t_{m}+t_{\mathrm{nm}}$ are much less than $\lambda_{s d}$. Once $t_{m}+t_{\mathrm{nm}} \gtrsim \lambda_{s d}$, one goes over to $\operatorname{CPP}(2)$; as $t_{m}+t_{\mathrm{nm}}=d_{\mathrm{in}} \gg \lambda_{\mathrm{mfp}}$, one is in the local limit and, as mentioned earlier the magnetoresistance goes to zero. In other words, the CPP-MR maintains its plateau in Fig. 7, only as long as $d_{\text {in }} \ll \lambda_{s d}$.

Although the electron mean free path was crucial to understanding the dependence of the CIP-MR on $t_{\mathrm{nm}}$ and $t_{m}$, this is not the case for CPP. Here the only relevant length scale is the spin-diffusion distance $\lambda_{s d}$. As it is typically an order of magnitude larger than $\lambda_{\mathrm{mfp}}$, it does not seem to play a role in the interpretation of the CPP$\mathrm{MR}$ results in $\mathrm{Co} / \mathrm{Ag}$ and $\mathrm{Co} / \mathrm{Cu}^{2}$

\section{SUMMARY}

We have shown that in CPP there are two distinct possibilities depending on whether the currents associated with spin-up and spin-down electrons mix or not. If they do, which occurs when the layer repeat distance $d_{\text {in }}$ is large compared to the spin-diffusion distance $\lambda_{s d}$, the spin is no longer a good quantum number for the currents, the two currents mix and one loses the giant magnetoresistive effect that is due to a "short-circuit" effect for one of the currents. ${ }^{1}$ When $\lambda_{s d} \gg d_{\text {in }}$ the currents do not mix, and as the electron mean free path $\lambda_{\mathrm{mfp}}$ does not cause the magnetoresistance to decrease for the CPP geometry, we find reasonably large magnetoresistances extending out to large bilayer repeat distances.

\section{ACKNOWLEDGMENTS}

We thank Professor Albert Fert, Professor William Pratt, Jr., and Professor Peter Schroeder for very helpful discussions. This research was supported by the Office of Naval Research Grant No. N00014-91-J-1695, and the New York University Technology Transfer Fund.
${ }^{1}$ S. Zhang, P. M. Levy, and A. Fert, Phys. Rev. B 45, 8689 (1992).

${ }^{2}$ W. P. Pratt, Jr., S.-F. Lee, J. M. Slaughter, R. Loloee, P. A. Schroeder, and J. Bass, Phys. Rev. Lett. 66, 3060 (1991); P. A. Schroeder et al. (unpublished).

${ }^{3}$ G. D. Mahan, Many-Particle Physics (Plenum, New York, 1981), pp. 185-196.

${ }^{4}$ Actually, it follows directly from the Kubo formula that $\nabla \cdot \sigma\left(\mathbf{r}, \mathbf{r}^{\prime}\right)=0$, and $\sigma\left(\mathbf{r}, \mathbf{r}^{\prime}\right) \cdot \stackrel{\leftarrow}{\nabla^{\prime}}=0$ (with the gradient operator acting to the left), and the equation of continuity is trivially satisfied, independently of the electric-field distribution $\mathbf{E}\left(\mathbf{r}^{\prime}\right)$; see C. L. Kane, R. A. Serota, and P. A. Lee, Phys. Rev. B 37, 6701 (1988). We would like to thank Professor Sadamichi Maekawa for bringing this reference to our attention. This is true if one calculates the total two-point conductivity. Instead, in this paper we only consider the subset of diagrams that corresponds to the short-range part (governed by the elastic mean free paths) and that yields the Boltzmann conductivity in the limit $k_{F} \lambda \gg 1$ [see $\mathrm{H}$. E. Camblong and P. M. Levy, Phys. Rev. Lett. 69, 2835 (1992)]. Actually, there exist extra ladder diagrams (which we have neglected) that lead to a long-range contribution associated with diffusion processes and such that the total conductivity is divergenceless. However, the global conductance calculated with our short-range conductivity and fields subject to the constraints (25) is the same as the true global conductance calculated with the total conductivity but with arbitrary fields. These results will be discussed in a forthcoming publication.

${ }^{5}$ S. Zhang and P. M. Levy, J. Appl. Phys. 69, 4786 (1991). ${ }^{6}$ S. Zhang, H. E. Camblong, and P. M. Levy (unpublished). ${ }^{7}$ S. Zhang and P. M. Levy, in Magnetic Surfaces, Thin Films and Multilayers, edited by S. S. Parkin et al., MRS Symposia Proceedings No. 231 (Materials Research Society, Pittsburgh, 1992), p. 255. 